\title{
Measuring Out-of-Time-Order Correlators on a Nuclear Magnetic Resonance Quantum Simulator
}

\author{
Jun Li, ${ }^{1}$ Ruihua Fan, ${ }^{2,3}$ Hengyan Wang, ${ }^{3}$ Bingtian Ye, ${ }^{3}$ Bei Zeng, ${ }^{4,5,2,{ }^{*}}$ Hui Zhai, ${ }^{2,6, \dagger}$ Xinhua Peng, ${ }^{7,8,9, *}$ and Jiangfeng Du ${ }^{7,8}$ \\ ${ }^{1}$ Beijing Computational Science Research Center, Beijing 100193, China \\ ${ }^{2}$ Institute for Advanced Study, Tsinghua University, Beijing 100084, China \\ ${ }^{3}$ Department of Physics, Peking University, Beijing 100871, China \\ ${ }^{4}$ Department of Mathematics and Statistics, University of Guelph, Guelph N1G 2W1, Ontario, Canada \\ ${ }^{5}$ Institute for Quantum Computing, University of Waterloo, Waterloo N2L 3G1, Ontario, Canada \\ ${ }^{6}$ Collaborative Innovation Center of Quantum Matter, Beijing 100084, China \\ ${ }^{7}$ Hefei National Laboratory for Physical Sciences at Microscale and Department of Modern Physics, \\ University of Science and Technology of China, Hefei, Anhui 230026, China \\ ${ }^{8}$ Synergetic Innovation Centre of Quantum Information and Quantum Physics, \\ University of Science and Technology of China, Hefei, Anhui 230026, China \\ ${ }^{9}$ College of Physics and Electronic Science, Hubei Normal University, Huangshi, Hubei 435002, China
}

(Received 30 December 2016; published 19 July 2017)

\begin{abstract}
The idea of the out-of-time-order correlator (OTOC) has recently emerged in the study of both condensed matter systems and gravitational systems. It not only plays a key role in investigating the holographic duality between a strongly interacting quantum system and a gravitational system, it also diagnoses the chaotic behavior of many-body quantum systems and characterizes information scrambling. Based on OTOCs, three different concepts-quantum chaos, holographic duality, and information scrambling - are found to be intimately related to each other. Despite its theoretical importance, the experimental measurement of the OTOC is quite challenging, and thus far there is no experimental measurement of the OTOC for local operators. Here, we report the measurement of OTOCs of local operators for an Ising spin chain on a nuclear magnetic resonance quantum simulator. We observe that the OTOC behaves differently in the integrable and nonintegrable cases. Based on the recent discovered relationship between OTOCs and the growth of entanglement entropy in the many-body system, we extract the entanglement entropy from the measured OTOCs, which clearly shows that the information entropy oscillates in time for integrable models and scrambles for nonintgrable models. With the measured OTOCs, we also obtain the experimental result of the butterfly velocity, which measures the speed of correlation propagation. Our experiment paves a way for experimentally studying quantum chaos, holographic duality, and information scrambling in many-body quantum systems with quantum simulators.
\end{abstract}

DOI: 10.1103/PhysRevX.7.031011

\section{INTRODUCTION}

The out-of-time-order correlator (OTOC), given by

$$
F(t)=\left\langle\hat{B}^{\dagger}(t) \hat{A}^{\dagger}(0) \hat{B}(t) \hat{A}(0)\right\rangle_{\beta},
$$

is proposed as a quantum generalization of a classical measure of chaotic behaviors $[1,2]$. Here, $\hat{H}$ is the system

\footnotetext{
*zengb@uoguelph.ca

†hzhai@tsinghua.edu.cn

*xhpeng@ustc.edu.cn
}

Published by the American Physical Society under the terms of the Creative Commons Attribution 4.0 International license. Further distribution of this work must maintain attribution to the author(s) and the published article's title, journal citation, and DOI.
Subject Areas: Quantum Physics,

Quantum Information,

Statistical Physics
Hamiltonian, $\hat{B}(t)=e^{i \hat{H} t} \hat{B} e^{-i \hat{H} t}$, and $\langle\cdots\rangle_{\beta}$ denotes averaging over a thermal ensemble at the temperature $1 / \beta=k_{B} T$. For a many-body system with local operators $\hat{A}$ and $\hat{B}$, the exponential deviation from unity of a normalized OTOC, i.e., $F(t) \sim 1-\# e^{\lambda_{L} t}$, gives rise to the Lyapunov exponent $\lambda_{L}$.

Quite remarkably, it was found recently that the OTOC also emerges in a different system that seems unrelated to chaos, that is, the scattering of shock waves nearby the horizon of a black hole and the information scrambling there [3-5]. A Lyapunov exponent of $\lambda_{L}=2 \pi / \beta$ is found there. Later it was also found that the quantum correction from string theory always makes the Lyapunov exponent smaller [5]. Thus, it leads to a conjecture that $2 \pi / \beta$ is an upper bound of the Lyaponuv exponent, which was later proved for generic quantum systems [6]. This is a profound 
theoretical result. If a quantum system is exactly holographic dual to a black hole, its Lyapunov exponent will saturate the bound; and a more nontrivial speculation is that if the Lyapunov exponent of a quantum system saturates the bound, it will possess a holographic dual to a gravity model with a black hole. A concrete quantum mechanics model, now known as the Sachdev-Ye-Kitaev model, has been shown to fulfill this conjecture $[2,7,8]$. This establishes a profound connection between the existence of holographic duality and the chaotic behavior in many-body quantum systems [9].

Recent studies also reveal that the OTOC can be applied to study physical properties beyond chaotic systems. The decay of the OTOC is closely related to the delocalization of information and implies the information-theoretic definition of scrambling. In the high- temperature limit (i.e., $\beta=0$ ), a connection between the OTOC and the growth of entanglement entropy in quantum many-body systems has also been discovered quite recently $[10,11]$. The OTOC can also characterize many-body localized phases, which are not even thermalized [10,12-15].

Despite the significance of the OTOC revealed by recent theories, experimental measurement of the OTOC remains challenging. First of all, unlike the normal time-ordered correlators, the OTOC cannot be related to conventional spectroscopy measurements, such as angle-resolved photoemission spectroscopy (ARPES) and neutron scattering, through the linear response theory. Secondly, direct simulation of this correlator requires the backward evolution in time, that is, the ability to completely reverse the Hamiltonian, which is extremely challenging. One experimental approach closely related to time reversal of quantum systems is the echo technique [16], and the echo has been studied extensively for both noninteracting particle systems and many-body systems to characterize the stability of quantum evolution in the presence of perturbations [17-19], and the physics is already quite close to OTOC. Recently it has been proposed that the OTOC can be measured using echo techniques [20]. In addition, there also exists several other theoretical proposals based on the interferometric approaches [21-23]. However, none of them have been experimentally implemented thus far.

Here, we adopt a different approach to measure the OTOC. To make our approach work, some extent of "local control" is required. A universal quantum computer fulfills this need by having "full local control" of the system-that is, a universal set of local evolutions can be realized, and this set of local evolutions can build up any unitary evolution of the many-body system, both forward and backward evolution in time. That is to say, we use a quantum computer to perform the measurement of the OTOC. In fact, historically, one of the key motivations to develop quantum computers is to simulate the dynamics of many-body quantum systems [24], and quantum simulation of many-body dynamics has been theoretically shown to be

efficient with practical algorithms proposed [25]. Here, the quantum computer we use is liquid-state nuclear magnetic resonance (NMR) with molecules. In this work, we report measurements of OTOCs on a NMR quantum simulator. We stress that, on one hand, our approach is universal and can be applied to any system that has full local quantum control, including a superconducting qubit and trapped ion; on the other hand, this experiment is currently limited to a small size not because of our scheme but because of the scalability issue of the quantum computer.

\section{NMR QUANTUM SIMULATION OF THE OTOC}

The system we simulate is an Ising spin chain model, whose Hamiltonian is written as

$$
\hat{H}=\sum_{i}\left(-\hat{\sigma}_{i}^{z} \hat{\sigma}_{i+1}^{z}+g \hat{\sigma}_{i}^{x}+h \hat{\sigma}_{i}^{z}\right),
$$

where $\hat{\sigma}_{i}^{x, y, z}$ are Pauli matrices on the $i$ site. The parameter values $g=1, h=0$ correspond to the traverse field Ising model, where the system is integrable. The system is nonintegrable whenever both $g$ and $h$ are nonzero. We simulate the dynamics governed by the system Hamiltonian $\hat{H}$, and measure the OTOCs of operators that are initially acting on different local sites. The time dynamics of the OTOCs are observed, from which entanglement entropy of the system and butterfly velocities of the chaotic systems are extracted.

\section{A. Physical system}

The physical system to perform the quantum simulation is the ensemble of nuclear spins provided by iodotrifluroethylene $\left(\mathrm{C}_{2} \mathrm{~F}_{3} \mathrm{I}\right)$, which is dissolved in $d$ chloroform; see Fig. 1(a) for the sample's molecular structure. For this
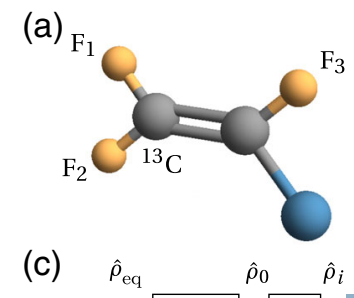

(b)

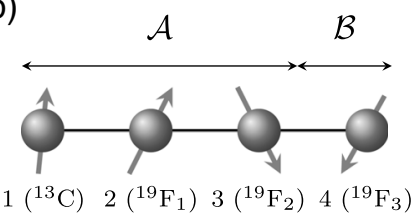

$\begin{array}{lll}\hat{\rho}_{\mathrm{eq}} & \hat{\rho}_{0} & \hat{\rho}_{i}\end{array}$

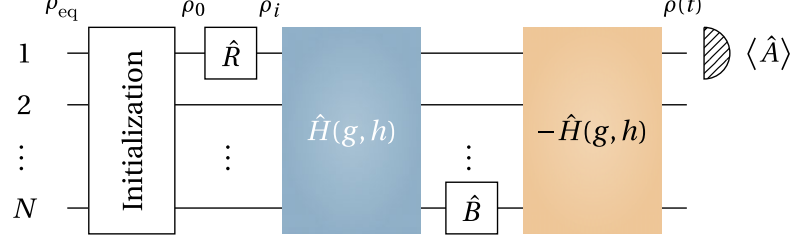

FIG. 1. Illustration of the physical system, the Ising model, and the experimental scheme. (a) The structure of the $\mathrm{C}_{2} \mathrm{~F}_{3} \mathrm{I}$ molecule used for the NMR simulation. (b) The four site Ising spin chain. $\mathcal{A}$ and $\mathcal{B}$ label two subsystems for the later discussion of the entanglement entropy. (c) Quantum circuit for measuring the OTOC for the general $N$-site Ising chain when $\beta=0$ (in our case, $N=4)$. Here, $\hat{R}=\mathbf{1}, \hat{R}_{x}(-\pi / 2), \hat{R}_{y}(\pi / 2)$ for $\hat{A}=\hat{\sigma}_{1}^{z}, \hat{\sigma}_{1}^{y}, \hat{\sigma}_{1}^{x}$, respectively. 
molecule, the ${ }^{13} \mathrm{C}$ nucleus and the three ${ }^{19} \mathrm{~F}$ nuclei $\left({ }^{19} \mathrm{~F}_{1}\right.$, ${ }^{19} \mathrm{~F}_{2}$, and ${ }^{19} \mathrm{~F}_{3}$ ) constitute a four-qubit quantum simulator. Each nucleus corresponds to a spin site of the Ising chain, as shown in Fig. 1(b). In experiment, the sample is placed in a static magnetic field along the $\hat{z}$ direction, resulting in the following form of the system Hamiltonian,

$$
\hat{H}_{\mathrm{NMR}}=-\sum_{i=1}^{4} \frac{\omega_{0 i}}{2} \hat{\sigma}_{i}^{z}+\sum_{i<j,=1}^{4} \frac{\pi J_{i j}}{2} \hat{\sigma}_{i}^{z} \hat{\sigma}_{j}^{z},
$$

where $\omega_{0 i} / 2 \pi$ is the Larmor frequency of spin $i$ and $J_{i j}$ is the coupling strength between spins $i$ and $j$. The values of these system parameters are given in Appendix A. The system is controlled by radio-frequency (rf) pulses, and the corresponding control Hamiltonian is

$$
\hat{H}_{\mathrm{rf}}(t)=-\omega_{1}(t)\left\{\cos [\phi(t)] \hat{\sigma}_{i}^{x}+\sin [\phi(t)] \hat{\sigma}_{i}^{y}\right\},
$$

where $\omega_{1}(t)$ and $\phi(t)$ denote the amplitude and the emission phase of the rf field, respectively. The control pulse shape can be elaborately monitored to realize the desired dynamic evolution. Actually, complete controllability of such a system has been demonstrated [26], which guarantees that arbitrary system evolution can be implemented on it. Our experiments are carried out on a Bruker AV $400 \mathrm{MHz}$ spectrometer $(9.4 \mathrm{~T})$ at temperature $T=305 \mathrm{~K}$.

\section{B. Experimental procedure}

As schematically illustrated in Fig. 1(c), here we focus on the $\beta=0$ case, and measuring the OTOC mainly consists of the following parts.

1. Initial state preparation. This step aims at preparing an initial state with density matrix $\hat{\rho}_{i} \propto \hat{A}=\hat{\sigma}_{1}^{\alpha}, \alpha=x, y$, or $z$.

1.1. The natural system is originally in the thermal equilibrium state $\hat{\rho}_{\text {eq }}$ populated according to the Boltzmann distribution. In the high-temperature approximation, $\hat{\rho}_{\mathrm{eq}} \approx 1 / 2^{4}\left(\mathbf{1}+\sum_{i=1}^{4} \epsilon_{i} \hat{\sigma}_{i}^{z}\right)$, where $\mathbf{1}$ is the identity and $\epsilon_{i} \sim 10^{-5}$ denotes the equilibrium polarization of spin $i$. Because there is no observable and unitary dynamical effect on $\mathbf{1}$, effectively we write $\hat{\rho}_{\text {eq }}=\sum_{i=1}^{4} \epsilon_{i} \hat{\sigma}_{i}^{z}$.

1.2. We engineer the system from $\hat{\rho}_{\text {eq }}$ into $\hat{\rho}_{0}=\sigma_{1}^{z}$. This is accomplished in two steps: first we remove the polarizations of the spins except for that of $\mathrm{F}_{2}$ by using selective saturation pulses, and then we transfer the polarization from $\mathrm{F}_{2}$ to ${ }^{13} \mathrm{C}$. Details of the method are described in Appendix B.

1.3. For the initial state $\hat{\rho}_{0}$ with $\alpha=x, y$, we need to further rotate the spin at site 1 by a $\pi / 2$ pulse around the $y$ or $-x$ axes, respectively.

2. Implementation of unitary evolution of $\hat{U}(t)=$ $e^{i \hat{H} t} \hat{B} e^{-i \hat{H} t}$. The key point is that according to the Trotter formula [25], the time evolution $e^{-i \hat{H} t}$ of the Ising spin chain of Eq. (2) can be approximately simulated through the decomposition

$$
e^{-i \hat{H} m \tau} \approx\left(e^{-i \hat{H}_{x} \tau / 2} e^{-i \hat{H}_{z} \tau / 2} e^{-i \hat{H}_{z z} \tau} e^{-i \hat{H}_{z} \tau / 2} e^{-i \hat{H}_{x} \tau / 2}\right)^{m}
$$

for small enough $\tau$. Here, the dynamics is divided into $m$ pieces with $t=m \tau$, and

$$
\begin{aligned}
\hat{H}_{x} & =\sum_{i} g \hat{\sigma}_{i}^{x}, \\
\hat{H}_{z} & =\sum_{i} h \hat{\sigma}_{i}^{z}, \\
\hat{H}_{z z} & =\sum_{i}-\hat{\sigma}_{i}^{z} \hat{\sigma}_{i+1}^{z} .
\end{aligned}
$$

Each propagator inside the bracket of Eq. (5) corresponds to either single-spin operation or coupled two-spin operation, and can be implemented through manipulating $\hat{H}_{\mathrm{NMR}}$ with rf control $\hat{H}_{\mathrm{rf}}$ : single-spin operation terms are global rotations around the $x$ or $z$ axis, which can be easily done through hard pulses; the two-spin operation term $e^{-i \hat{H}_{z z} \tau}$ can be generated through some suitably designed pulse sequence based on the NMR refocusing techniques [27]. More details of the method are described in Appendix B. The reversal of Ising dynamics $e^{i \hat{H} t}$ can be done in a similar manner. Note that in the case we consider here, $\hat{B}$ is a local unitary operator on the site- $N$ spin and $\hat{B}=\hat{\sigma}_{N}^{\gamma}$ with $\gamma=x$, $y, z$ that can be implemented by a selective if $\pi$ pulse on the site- $N$ spin. Hence, for any given $t$, the total unitary evolution $e^{i \hat{H} t} \hat{B} e^{-i \hat{H} t}$ can be simulated.

3. Readout. The OTOC is obtained by measuring the expectation value of the observable $\hat{O}=$ $e^{i \hat{H} t} \hat{B} e^{-i \hat{H} t} \hat{A} e^{i \hat{H} t} \hat{B} e^{-i \hat{H} t} \hat{A}$. For the infinite temperature $\beta=0$, the equilibrium state of the many-body system $\hat{H}$ is the maximally mixed state $1 / 2^{4}$. Since

$$
\langle\hat{O}\rangle_{\beta=0}=\operatorname{Tr}\left[\hat{U}(t) \hat{\rho}_{0} \hat{U}^{\dagger}(t) \hat{A}\right],
$$

when $\hat{B}$ is unitary, $\hat{U}(t) \hat{\rho}_{0} \hat{U}^{\dagger}(t)$ is a density matrix $\rho(t)$ evolved from $\rho_{0}$ by $\hat{U}(t)$, as simulated in step 2 . Finally, measuring the expectation value of $\hat{A}$ under $\rho(t)$ gives $\langle\hat{O}\rangle_{\beta=0}$. Because the NMR detection is performed on a bulk ensemble of molecules, readout is an ensembleaveraged macrosopic measurement. When the system is prepared at state $\rho(t)$, the expectation value of $\hat{A}$ can then be directly obtained from the spectrum. See Appendix B for details.

\section{Results of OTOC}

Two sets of typical experimental results of the OTOC at $\beta=0$ are shown in Fig. 2. Here, we normalize the OTOC by $\left\langle\hat{B}^{\dagger}(0) \hat{B}(0)\right\rangle\left\langle\hat{A}^{\dagger}(0) \hat{A}(0)\right\rangle$, and because $\hat{A}$ and $\hat{B}^{\dagger}$ commute at $t=0$, the initial value of this normalized OTOC is unity. The experimental data (red points) agree very well 
(a)

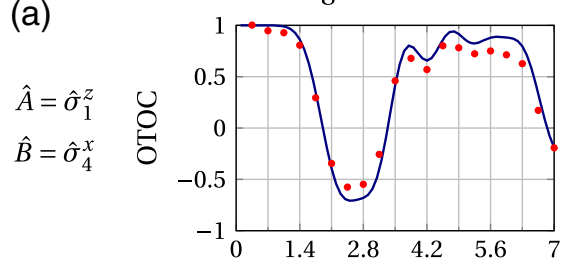

(b)

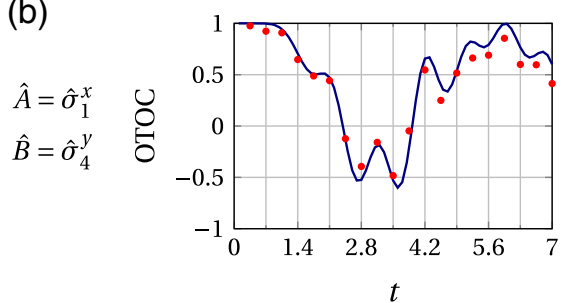

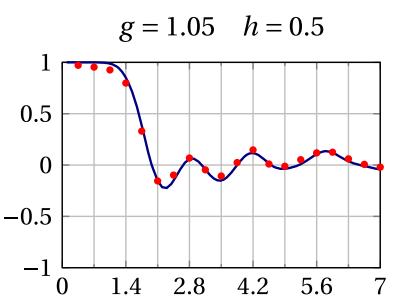
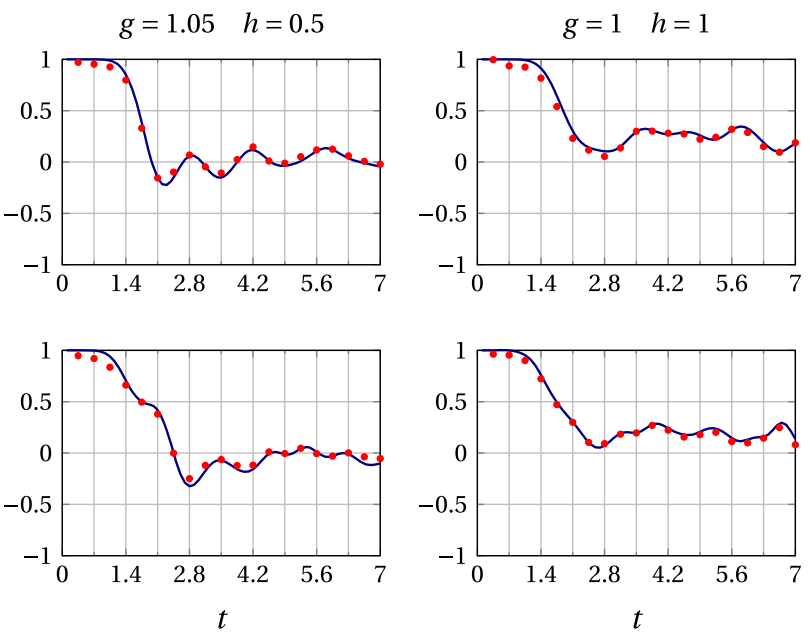

FIG. 2. Experimental results of OTOC measurement for an Ising spin chain. (a) $\hat{A}=\hat{\sigma}_{1}^{z}$ at the first site and $\hat{B}=\hat{\sigma}_{4}^{x}$ at the fourth site. (b) $\hat{A}=\hat{\sigma}_{1}^{x}$ at the first site and $\hat{B}=\hat{\sigma}_{4}^{y}$ at the fourth site. The three columns correspond to $g=1, h=0 ; g=1.05, h=0.5$; and $g=1$, $h=1$ of model Eq. (2), respectively. The red points are experimental data, the blue curves are theoretical calculation of OTOC with model Eq. (2) for four sites.

with the theoretical results (blue curves). The sources of experimental errors include imperfections in state preparation, control inaccuracy, and decoherence. See Appendix $\mathrm{C}$ for more details. We also measure OTOC for other operators $\left(\hat{A}=\hat{\sigma}_{1}^{\alpha}, \hat{B}=\hat{\sigma}_{4}^{\gamma}\right.$, with $\left.\alpha, \gamma=x, y, z\right)$ and they all behave similarly. The experimental results are in Appendix B.

In both the integrable case (the first column in Fig. 2) and the nonintegrable cases (the second and third columns in Fig. 2), the early time behaviors look similar. That is, the OTOC starts to deviate from unity after a certain time (for the unit of time $t$, see Appendix D for details.). However, the long time behaviors are very different between the integrable and nonintegrable cases. In the integrable case, after the decreasing period, the OTOC revives and recovers unity. This reflects that the system has a well-defined quasiparticle. And there exists an extensive number of integrals of motion, which is related to the fact that an integrable system does not thermalize. While in the nonintegrable case, the OTOC decreases to a small value and oscillates, which will not revive back to unity in a practical time scale. This relates to the fact that the information does scramble in a nonintegrable system [11].

\section{ENTROPY DYNAMICS}

To better illustrate the different behaviors of the information dynamics in the two cases of integrable and nonintegrable systems, we reconstruct the entanglement entropy of a subsystem from the measured OTOCs. Entanglement entropy has become an important quantity not only for quantum information processing but also for describing a quantum many-body system, such as quantum phase transition, topological order, and thermalization.
However, measuring entanglement entropy is always challenging [28].

The OTOC opens a new door for entropy measurement. An equivalence relationship between OTOCs at equilibrium and the growth of the second Rényi entropy after a quench has recently been established [10], which states that

$$
\exp \left(-S_{\mathcal{A}}^{(2)}\right)=\sum_{\hat{M} \in \mathcal{B}}\langle\hat{M}(t) \hat{V}(0) \hat{M}(t) \hat{V}(0)\rangle_{\beta=0}
$$

In the left-hand side of Eq. (8), $S_{\mathcal{A}}^{(2)}$ is the second Rényi entropy of the subsystem $\mathcal{A}$, after the system is quenched by an operator $\hat{O}$ at time $t=0$. That is, $S_{\mathcal{A}}^{(2)}=-\log \hat{\rho}_{\mathcal{A}}^{2}$ and $\hat{\rho}_{\mathcal{A}}=\operatorname{Tr}_{\mathcal{B}}\left(e^{-i \hat{H} t} V e^{i \hat{H} t}\right)$, and $\hat{V}=\hat{O} \hat{O}^{\dagger}$, up to a certain normalization condition (see Appendix E). The right-hand side of Eq. (8) is a summation over OTOCs at equilibrium. $\hat{M}$ is a complete set of operators in the subsystem $\mathcal{B}$.

In our experiment, we choose the quench operator $\hat{O} \propto$ $\left(\mathbf{1}+\hat{\sigma}_{1}^{x}\right)$ at the first site, and we take the first three sites as the subsystem $\mathcal{A}$ and the fourth site as the subsystem $\mathcal{B}$, as marked in Fig. 1(b). In this setting, $S_{\mathcal{A}}^{(2)}$ measures how much the quench operation induces additional correlation between the subsystems $\mathcal{A}$ and $\mathcal{B}$.

We take a complete set of operators in the subsystems $\mathcal{B}$ as $\hat{\sigma}_{4}^{\alpha}$ (up to a normalization factor), where $\alpha=0, x, y, z$ and $\hat{\sigma}^{0}=\mathbf{1}$. Since $\hat{V}=\hat{O} \hat{O}^{\dagger} \propto\left(\mathbf{1}+\hat{\sigma}_{1}^{x}\right)$, the right-hand side of Eq. (8) becomes a set of OTOCs that are given by

$$
\left\langle\hat{\sigma}_{4}^{\alpha}(t)\left(\mathbf{1}+\hat{\sigma}_{1}^{x}\right) \hat{\sigma}_{4}^{\alpha}(t)\left(\mathbf{1}+\hat{\sigma}_{1}^{x}\right)\right\rangle_{\beta=0} .
$$

Notice that $\operatorname{Tr}\left[\hat{\sigma}_{4}^{\alpha}(t) \hat{\sigma}_{1}^{x} \hat{\sigma}_{4}^{\alpha}(t)\right]=\operatorname{Tr}\left[\hat{\sigma}_{4}^{\alpha}(t) \hat{\sigma}_{4}^{\alpha}(t) \hat{\sigma}_{1}^{x}\right]=0$; the nonzero terms in Eq. (9) are nothing but OTOCs with $\hat{B}=$ $\hat{\sigma}_{4}^{\alpha}(\alpha=x, y, z)$ and $\hat{A}=\hat{\sigma}_{1}^{x}$, which are exactly what we 


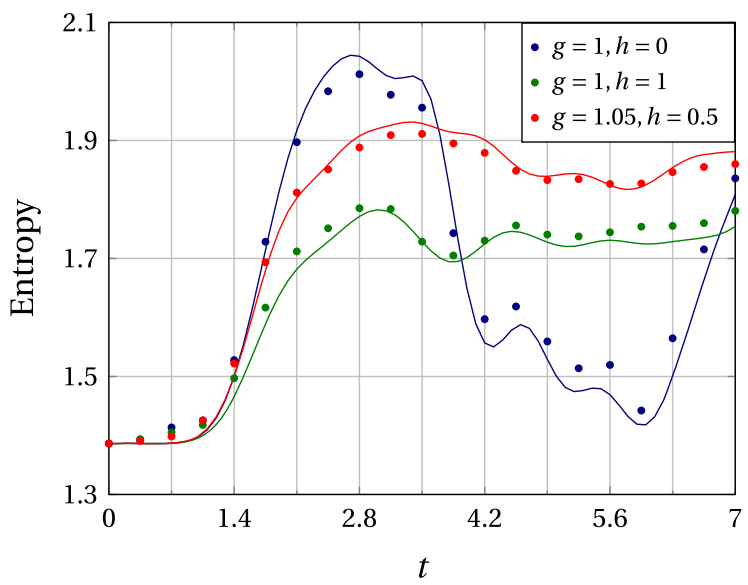

FIG. 3. The second Rényi entropy $S_{\mathcal{A}}^{(2)}$ after a quench. A quench operator $\left(\mathbf{1}+\hat{\sigma}_{1}^{x}\right)$ (up to a normalization factor) is applied to the system at $t=0$, and the entropy is measured by tracing out the fourth site as the subsystem $\mathcal{B}$. Different colors correspond to different parameters of $g$ and $h$ in the Ising spin model. The points are experimental data, the curves are theoretical calculations.

measure. That is to say, with the help of the relationship between OTOCs and entanglement growth, we can extract the growth of the entanglement entropy after the quench from the experimental data.

The results of the second Rényi entropy $S_{\mathcal{A}}^{(2)}$ are shown in Fig. 3. At short time, all three curves start to grow significantly after a certain time. This demonstrates that it takes a certain time for the perturbation applied at the first site to propagate to the subsystem $\mathcal{B}$ at the fourth site (see the discussion of butterfly velocity below). Then, for all

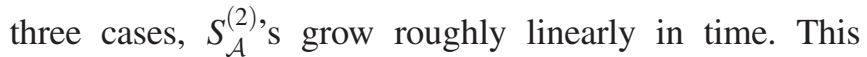
indicates that the extra information caused by the initial quench starts to scramble between subsystems $\mathcal{A}$ and $\mathcal{B}$. The differences lie in the long-time regime. For the integrable model, the $S_{\mathcal{A}}^{(2)}$ oscillates back to around its initial value after some time, which means that this extra information moves back to the subsystem $\mathcal{A}$ around that time window. As a comparison, such a large amplitude oscillation does not occur for the two nonintegrable cases and the $S_{\mathcal{A}}^{(2)}$ s saturate after growing. This supports the physical picture that the local information moves around in the integrable model, while it scrambles in the nonintegrable models [11].

\section{BUTTERFLY VELOCITY}

The OTOC also provides a tool to determine the speed for correlation propagating. At $t=0, \hat{A}$ and $\hat{B}$ commute with each other since they are operators at different sites. As time grows, the higher-order terms in the BakerCampbell-Hausdorff formula,

$$
\hat{B}(t)=\sum_{k=0}^{\infty} \frac{(i t)^{k}}{k !}[H, \ldots,[H, B], \ldots],
$$
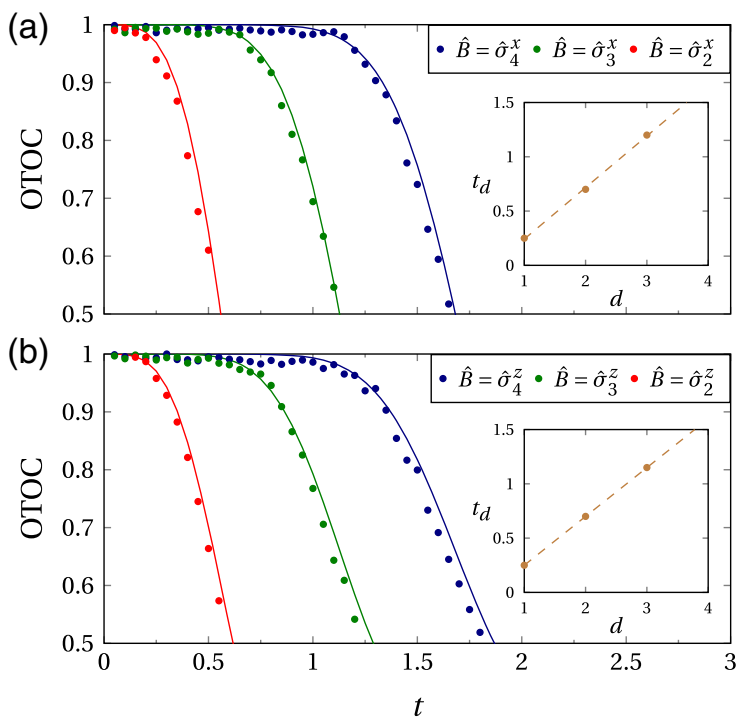

FIG. 4. Measurement of the butterfly velocity. (a) The OTOCs for $\hat{A}=\hat{\sigma}_{1}^{z}$ and $\hat{B}=\hat{\sigma}_{i}^{x}$, with $i=4$ (blue), $i=3$ (green), and $i=2$ (red). (b) The OTOCs for $\hat{A}=\hat{\sigma}_{1}^{y}$ and $\hat{B}=\hat{\sigma}_{i}^{z}$, with $i=4$ (blue), $i=3$ (green), and $i=2$ (red). The insets of (a) and (b) show the time for the onset of chaos $t_{d}$ for the OTOCs versus the distance between two operators. The slope gives $1 / v_{B}$. Here, $g=1.05$ and $h=0.5$.

become more and more important and some terms fail to commute with $\hat{A}$, at which the normalized OTOC starts to drop. Thus, the larger the distance between sites for $\hat{A}$ and $\hat{B}$, the later the time the OTOC starts deviating from unity. In general, the OTOC behaves as

$$
F(t)=a-b e^{\lambda_{L}\left(t-|x| / v_{B}\right)}+\cdots,
$$

where $a$ and $b$ are two nonuniversal constants and $|x|$ denotes the distance between two operators. Here, $v_{B}$ defines the butterfly velocity $[5,11,29-31]$. It quantifies the speed of a local operator growth in time and defines a light cone for chaos, which is also related to the LiebRobinson bound [31,32].

In our experiment, we fix $\hat{A}$ at the first site, and move $\hat{B}$ from the fourth site to the third site, and to the second site. From the experimental data, we can phenomenologically determine a characteristic time $t_{d}$ for the onset of chaos in each OTOC, i.e., the time that the OTOC starts departing from unity. By comparing the three different OTOCs in Fig. 4, it is clear that the closer the distance between $\hat{A}$ and $\hat{B}$, the smaller $t_{d}$. In the insets of Figs. 4(a) and 4(b), we plot $t_{d}$ as a function of the distance, and extract the butterfly velocity from the slope. We find that, for the OTOC with $\hat{A}=\hat{\sigma}_{1}^{z}$ and $\hat{B}=\hat{\sigma}_{i}^{x}, v_{B}=2.10$, and for the OTOC with $\hat{A}=\hat{\sigma}_{1}^{y}$ and $\hat{B}=\hat{\sigma}_{i}^{z}, v_{B}=2.22$. The butterfly velocity is nearly independent of the choice of local operators, which is a kind of manifestation of the chaotic behavior of the system. 


\section{OUTLOOK}

The OTOC provides a faithful reflection of the information scrambling and chaotic behavior of quantum many-body systems. It goes beyond the normal order correlators studied in linear response theory, which only capture the thermalization behavior of the system. Measuring the OTOC functions can reveal how quantum entanglement and information scrambles across all of the degrees of freedom in a system. In the future it will be possible to simulate more sophisticated systems that may possess holographic duality, with larger size and different $\beta$, to extract the corresponding Lyapunov exponents such that one can experimentally verify the connection between the upper bound of the Lyapunov exponent and the holographic duality.

We use liquid-state NMR as a quantum simulator for the demonstration of OTOC measurement. NMR provides an excellent platform to benchmark the measurement ideas and techniques. Our work here represents a first and encouraging step towards further experimentally observing OTOCs on large-sized quantum systems. The present method can be readily translated to other controllable systems. For instance, in trapped-ion systems, high-fidelity execution of arbitrary control with up to five atomic ions has been realized [33]. Superconducting quantum circuits also allow for engineering on local qubits with errors at or below the threshold [34,35], hence offering another very promising experimental approach. The progress in recent years in these two quantum hardware platforms has been fast and astounding, particularly in the pursuit of fabrication of quantum computing architecture at large scale. It is reckoned that quantum simulators consisting of tens of or even hundreds of qubits are within reach in the near future [36-39]. Experimentalists will see the great opportunity of applying these technologies for studying quantum chaotic behaviors for much more complicated quantum many-body systems.

\section{ACKNOWLEDGMENTS}

We thank Huitao Shen, Pengfei Zhang, Yingfei Gu, and Xie Chen for helpful discussions. B.Z. is supported by NSERC and CIFAR. H. Z. is supported by MOST (Grant no. 2016YFA0301604), Tsinghua University Initiative Scientific Research Program, and NSFC Grant No. 11325418. H. W., X. P., and J. D. would like to thank the following funding sources: NKBRP (2013CB921800 and 2014CB848700), the National Science Fund for Distinguished Young Scholars (11425523), and NSFC (11375167, 11227901, and 91021005). J. L. is supported by the National Basic Research Program of China (Grants No. 2014CB921403 and No. 2016YFA0301201), National Natural Science Foundation of China (Grants No. 11421063, No. 11534002, No. 11375167, and No. 11605005), the National Science Fund for Distinguished Young Scholars (Grant No. 11425523), and NSAF (Grant No. U1530401).

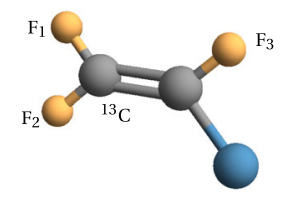

\begin{tabular}{|c|c|c|c|c|c|c|}
\hline & ${ }^{13} \mathrm{C}$ & $\mathrm{F}_{1}$ & $\mathrm{~F}_{2}$ & $\mathrm{~F}_{3}$ & $T_{2}^{*}(\mathrm{~s})$ & $T_{2}(\mathrm{~s})$ \\
\hline${ }^{13} \mathrm{C}$ & 15480.0 & & & & 1.22 & 7.9 \\
\hline $\mathrm{F}_{1}$ & -297.8 & -33131.7 & & & 0.66 & 4.4 \\
\hline $\mathrm{F}_{2}$ & -275.7 & 64.5 & -42682.7 & & 0.63 & 6.8 \\
\hline $\mathrm{F}_{3}$ & 39.3 & 51.4 & -129.0 & $\mid-56444.8$ & 0.61 & 4.8 \\
\hline
\end{tabular}

FIG. 5. Characteristics of iodotrifluroethylene. Molecular structure together with a table of the chemical shifts (on the diagonal) and $J$-coupling strengths (lower off diagonal), all in Hz. The chemical shifts are given with respect to base frequency for ${ }^{13} \mathrm{C}$ or ${ }^{19} \mathrm{~F}$ transmitters on the $400-\mathrm{MHz}$ spectrometer that we use.

Note added.-Recently, we noticed a related work [40], where OTOCs are measured in a trapped-ion quantum magnet.

\section{APPENDIX A: PARAMETERS OF THE SYSTEM HAMILTONIAN}

We use iodotrifluroethylene dissolved in $d$-chloroform [41]. The system Hamiltonian is given by

$$
\hat{H}_{\mathrm{NMR}}=-\sum_{i=1}^{4} \frac{\omega_{0 i}}{2} \hat{\sigma}_{i}^{z}+\sum_{i<j,=1}^{4} \frac{\pi J_{i j}}{2} \hat{\sigma}_{i}^{z} \hat{\sigma}_{j}^{z}
$$

where $\omega_{0 i} / 2 \pi$ is the Larmor frequency of $\operatorname{spin} i$, and $J_{i j}$ are the coupling strength between spins $i$ and $j$. The values of parameters $\omega_{0 i}$ and $J_{i j}$ are given in Fig. 5 .

\section{APPENDIX B: EXPERIMENTAL PROCEDURE}

\section{Initialization}

The system is required to be initialized into $\hat{\rho}_{0} \propto \hat{\sigma}_{1}^{z}$ from the equilibrium state $\hat{\rho}_{\text {eq }}$. We first exploit the steady-state effect when a relaxing nuclear spin system is subjected to multiple-pulse irradiation [42]. To implement this, we apply the periodic sequence $\left[\pi_{1,2,4}-d\right]$ to the system, where $\pi_{1,2,4}$ means simultaneous $\pi$ rotations on the spins ${ }^{13} \mathrm{C}_{,} \mathrm{F}_{1}$, and $\mathrm{F}_{3}$, and $d$ is a time delay parameter to be adjusted; see the first part of the circuit shown in Fig. 6(a). To do $\pi_{1,2,4}$, we use a pulse that is composed of three frequency components, each Hermite-180 shaped in 500 segments, with a duration of $1 \mathrm{~ms}$. With increasing the number of applied cycles, under the joint effects of relaxation and $\pi$ reversions, the equilibrium Zeeman magnetizations $\left\langle\hat{\sigma}_{1,2,4}^{z}\right\rangle$ gradually decay to zero. Only the magnetization $\hat{\sigma}_{3}^{z}$ is retained at last as it is the fixed point to the periodic driving. We adjust the time interval $d$ between the $\pi$ pulses to achieve the best-quality steady state. In experiment, we set $d=25 \mathrm{~ms}$ and after more than 500 cycles we find that the system is effectively steered into a steady state $\hat{\rho}_{s s} \propto \hat{\sigma}_{3}^{z}$ (in this sample, we do not see observable Overhauser enhancement). Next, with a SWAP operation we transfer the polarization from the 


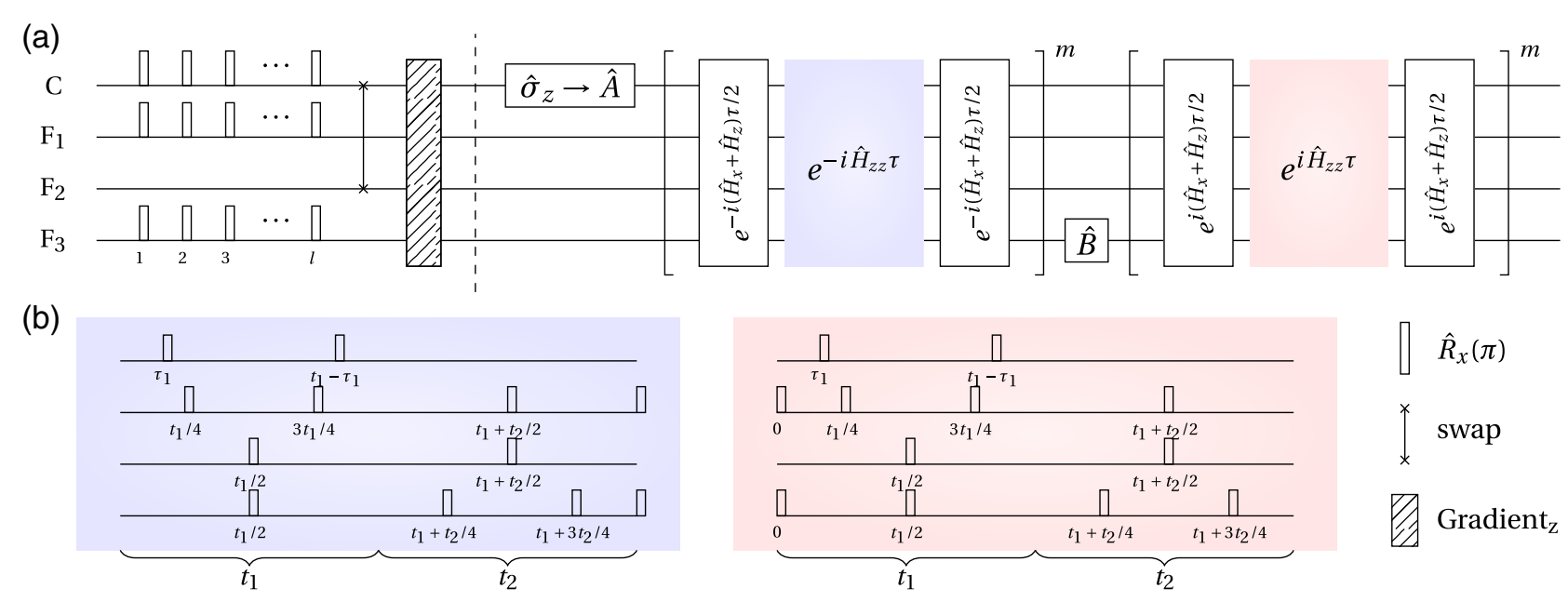

(c)

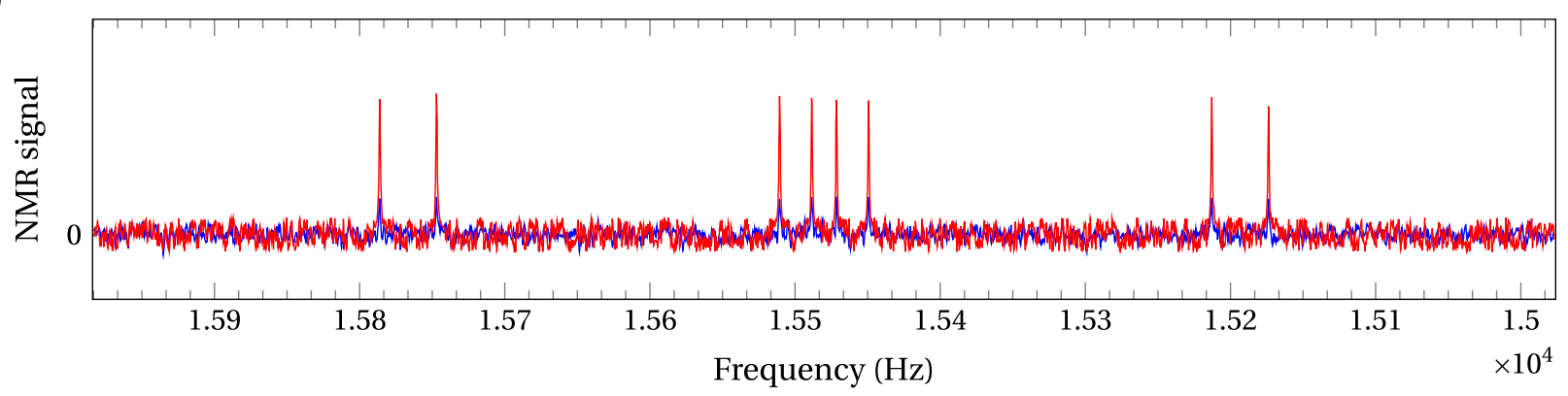

FIG. 6. (a) Quantum circuit that measures the OTOCs. The first part aims to reset an arbitrary state to the desired initial state. Here, the time interval between the $\pi$ pulses is $25 \mathrm{~ms}$, the number of cycles is $l=500$, and $G_{z}$ denotes $z$ axis gradient pulse. (b) Sequences for implementing the dynamics of $e^{-i \hat{H}_{z z} \tau}$ (left) and $e^{i \hat{H}_{z z} \tau}$ (right). The refocusing circuits are designed to generate the right amount of coupled evolution. (c) ${ }^{13} \mathrm{C}$ experimental spectrum for equilibrium state (blue) and state $\hat{\rho}_{0}$ (red) after a readout pulse $\hat{R}_{y}^{1}(\pi / 2)$. They are shown at the same scale for comparison.

high-sensitivity $F_{2}$ nucleus to the low-sensitivity ${ }^{13} \mathrm{C}$ nucleus. Using the method, we finally get an initial state $\hat{\rho}_{0} \propto \hat{\sigma}_{1}^{z}$. The resulting experimental spectrum is shown in Fig. 6(c).

\section{Simulating time evolution of Ising spin chain}

According to Eq. (5) of the main text, the key ingredient in simulating the evolution of Ising Hamiltonian $\hat{H}$ is to implement

$$
e^{-i \hat{H}_{x} \tau / 2} e^{-i \hat{H}_{z} \tau / 2} e^{-i \hat{H}_{z z} \tau} e^{-i \hat{H}_{z} \tau / 2} e^{-i \hat{H}_{x} \tau / 2}
$$

Here, except for $e^{-i \hat{H}_{z z} \tau}$, all of the other four terms are global rotation around the $x$ (and $z$ ) axis, which can be easily done through hard pulses. $e^{-i \hat{H}_{z z} \tau}$ can be generated by manipulating the natural physical Hamiltonian $\hat{H}_{\mathrm{NMR}}$ with a suitable refocusing scheme [43]. The basic idea is to evolve the system with the $J$ term in $\hat{H}_{\mathrm{NMR}}$ and then to use spin echoes to engineer the evolution. That is to say, for instance, for the $\hat{\sigma}_{i}^{z} \hat{\sigma}_{j}^{z}$ term, when a transverse $\pi$ pulse is applied to reverse the polarization of one of the two spins, the evolution is also reserved. Hence, by designing a suitable refocusing scheme, the dynamics of $\hat{H}_{z z}$ and $-\hat{H}_{z z}$ can be efficiently simulated.

Although a general and efficient refocusing scheme exists for any $\hat{\sigma}_{z} \hat{\sigma}_{z}$-coupled evolution [27], for the present task it is possible to find a much simplified circuit construction. Figure 6(b) shows our ideal circuits. Let $\mathrm{O}_{1}$ and $\mathrm{O}_{2}$ define the reference frequency for the carbon and fluorine channel, respectively. Consider the refocusing circuit [Fig. 6(b), left] for implementing $e^{-i \hat{H}_{z z} \tau}$; it automatically refocuses the fluorine spins and decouples the terms $J_{31}, J_{41}$, and $J_{43}$, and the evolution of the other terms should fulfil the following requirements to yield the right amount of evolution:

$$
\begin{gathered}
\left(\omega_{01} / 2 \pi-\mathrm{O}_{1}\right)\left(4 \tau_{1}-t_{1}+t_{2}\right)=0, \\
\pi J_{21} / 2 \times 4 \tau_{1}=-\tau, \\
-\pi J_{32} / 2 \times t_{2}=-\tau, \\
\pi J_{43} / 2 \times t_{1}=-\tau .
\end{gathered}
$$


The solution to the above system of equations is given by $\mathrm{O}_{1}=\omega_{01} / 2 \pi=15480.0 \mathrm{~Hz}, \quad t_{1}=0.004935 \tau, \quad t_{2}=$ $0.009870 \tau$, and $\tau_{1}=0.000534 \tau$. As to the refocusing circuit for implementing $e^{i \hat{H}_{z z} \tau}$, we find that it suffices to just make slight changes to the circuit for $-e^{i \hat{H}_{z z} \tau}$, as shown in Fig. 6, and one can then reverse the dynamics of all terms.

Now, the whole network for implementing Ising dynamics is expressed in terms of single-spin rotations and evolution of $J$ terms in $\hat{H}_{\mathrm{NMR}}$. In practice, each singlespin rotation is realized through a selective rf pulse of Gaussian shape, with a duration of $0.5-1 \mathrm{~ms}$. We then conduct a compilation procedure to the sequence of selective pulses to eliminate the control imperfections caused by off-resonance and coupling effects up to the first order $[44,45]$. To further improve the control performance, we employ the gradient ascent pulse engineering (GRAPE) technique [46] on the complied sequences. Because that compilation procedure has the capability of directly providing a good initial start for subsequent gradient iteration, the GRAPE searching quickly finds high-performance pulse controls for the desired propagators. The obtained shaped pulses for different sets of Hamiltonian parameters $(g, h)$ all have numerical fidelities above 0.999 , and have been optimized with practical control field inhomogeneity taken into consideration.

The Ising dynamics to be simulated is discretized into 20 steps, with each time step of duration $\tau=0.35 \mathrm{~ms}$. Choosing different operators for $\hat{A}$ and $\hat{B}$, we experimentally measure the corresponding OTOC. All of the experimental results are given in Fig. 7. The theoretical trajectories are plotted for comparison. Although some
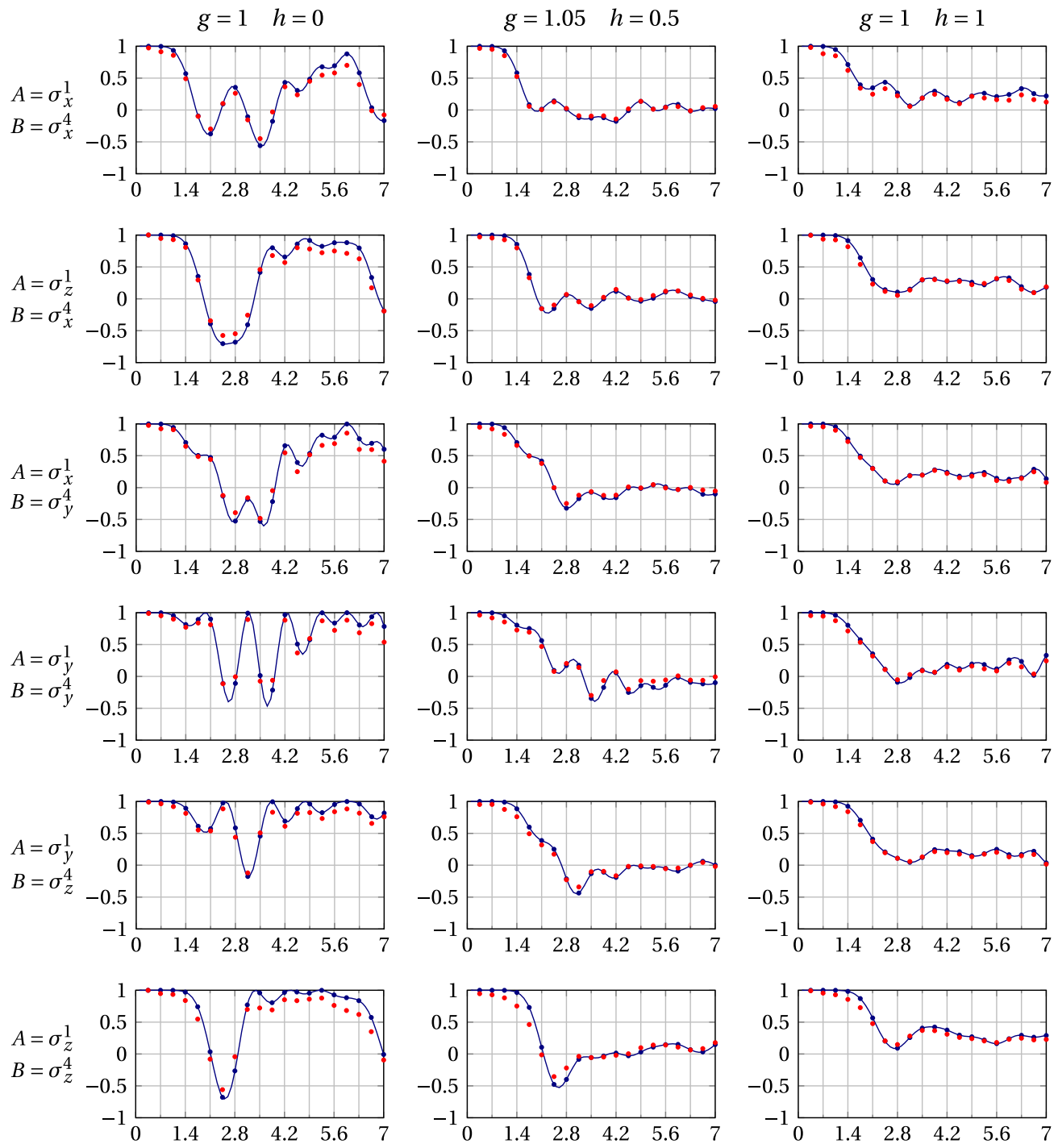

FIG. 7. Experimental results for measuring OTOCs for different Ising model parameters and different pairs of $\hat{A}$ and $\hat{B}$. The red points are experimental data, the blue curves are theoretical calculation of OTOCs with the model, and the blue points are theoretical values displayed for comparison. 
TABLE I. The standard deviations of $\langle\hat{A}\rangle$ for the experiments and numerical simulations when $\hat{A}=\hat{\sigma}_{1}^{x}, \hat{B}=\hat{\sigma}_{4}^{y}$.

\begin{tabular}{lccc}
\hline \hline & $g=1, h=1$ & $g=1.05, h=0.5$ & $g=1, h=0$ \\
\hline$\sigma_{\text {expt }}$ & 0.1097 & 0.0456 & 0.0308 \\
$\sigma_{\text {ini }}^{\text {err }}$ & 0.0340 & 0.0340 & 0.0340 \\
$\sigma_{\text {intromo }}^{\text {err }}$ & 0.0323 & 0.0150 & 0.0188 \\
$\sigma_{T_{2}}^{\text {err }}$ & 0.0461 & 0.0161 & 0.0214 \\
\hline \hline
\end{tabular}

discrepancies between the data and the simulations remain, the experimental results reflect very well how OTOCs behave differently in the integrable and chaotic cases.

\section{Readout}

All the observations are made on the probe spin ${ }^{13} \mathrm{C}$. Because we use an unlabeled sample in the real experiment, the molecules with a ${ }^{13} \mathrm{C}$ nucleus are present at a concentration of about $1 \%$. The NMR signal in the high field is obtained from the precessing transverse magnetization of the ensemble of molecules in the sample:

$$
\begin{aligned}
M(t) & =M_{x}(t)+i M_{y}(t) \\
& =\operatorname{Tr}\left[\hat{\rho}(t)\left(\sum_{j}\left\langle\sigma_{j}^{x}\right\rangle+i \sum_{j}\left\langle\sigma_{j}^{y}\right\rangle\right)\right] .
\end{aligned}
$$

As the precession frequencies of different spins are distinguishable, they can be individually detected; e.g., we obtain the measurements of $\left\langle\hat{\sigma}_{1}^{x}\right\rangle$ and $\left\langle\hat{\sigma}_{1}^{y}\right\rangle$ at the ${ }^{13} \mathrm{C}$ Larmor frequency. To measure $\left\langle\hat{\sigma}_{1}^{z}\right\rangle$, we need to apply a $\pi / 2$ rotation along $\hat{y}$. By fitting the ${ }^{13} \mathrm{C}$ spectrum, the real part and the imaginary parts of the peaks are extracted, which corresponds to $\left\langle\hat{\sigma}_{1}^{x}\right\rangle$ and $\left\langle\hat{\sigma}_{1}^{y}\right\rangle$, respectively.

\section{APPENDIX C: EXPERIMENTAL ERROR ANALYSIS}

The sources of experimental errors include imperfections in initial state preparation, infidelities of the GRAPE pulses, rf inhomogeneity, and decoherences. We make an analysis to the data set of the case $\hat{A}=\hat{\sigma}_{1}^{x}, \hat{B}=\hat{\sigma}_{4}^{y}$ to get an understanding of the role of each type of error source. We calculate the standard deviations $\sigma_{\text {expt }}:=\sqrt{\sum_{i=1}^{20}\left(\langle\hat{A}\rangle_{\text {expt }}^{i}-\langle\hat{A}\rangle_{\text {th }}^{i}\right)^{2} / 20}$ for the experimental data, which are presented in Table I.

We run the initialization process 50 times and find that the fluctuation of the initial state polarization of $\hat{\rho}_{0}$ is around $3.40 \%$. The fluctuation is due to (i) error in state preparation and (ii) error in spectrum fitting. The latter can be inferred from the signal-to-noise ratio of the spectrum, which is estimated to be $\approx 2.13 \%$.

All the GRAPE pulses for implementing $e^{-i \hat{H} \tau}$ and $e^{i \hat{H} \tau}$ are of fidelities above 0.999 . On such a precise level, if we assume no other sources of error and assume that the pulse generator ideally generates these pulses, then the experimental results should match the theoretical predictions almost perfectly.

Figure 8 plots the robustness of the GRAPE pulses in the presence of imperfections of $\mathrm{rf}$ fields in the ${ }^{13} \mathrm{C}$ channel and
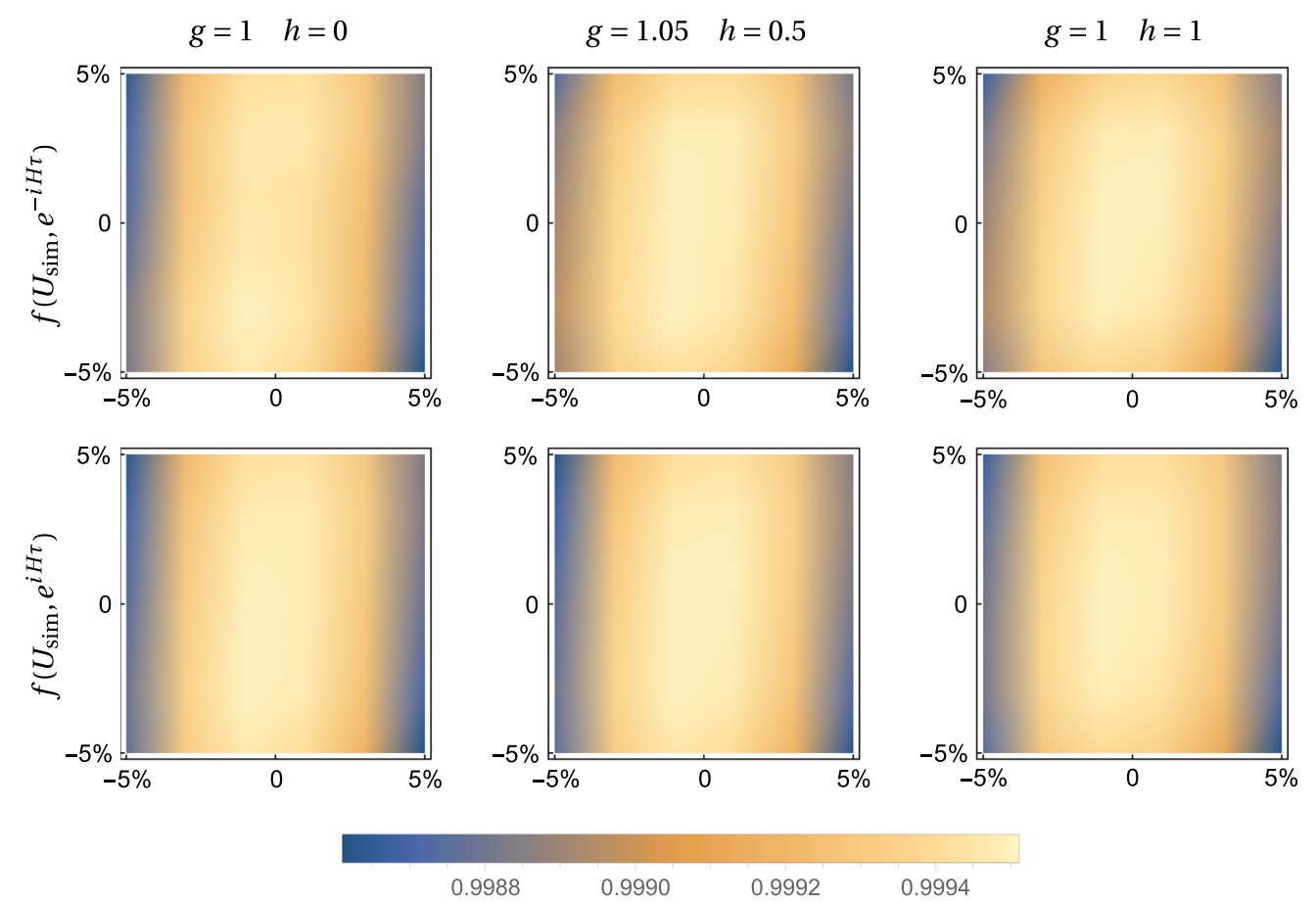

FIG. 8. Robustness of the used GRAPE pulses against rf field inhomogeneity. Here, the transverse axis denotes relative error of output field power of the ${ }^{13} \mathrm{C}$ channel and the longitudinal axis is that of the ${ }^{19} \mathrm{~F}$ channel. $U_{\text {sim }}$ denotes the corresponding propagator and $f$ is the fidelity function. 
${ }^{19} \mathrm{~F}$ channel. To understand to what extent the rf field inhomogeneity may affect the experimental results, we calculate the deviation of the dynamics based on a simple inhomogeneity model. The model assumes that the output power discrepancy of the rf fields is uniformly distributed between $\pm 3 \%$. The simulated results $\sigma_{\text {inhomo }}^{\text {err }}$ are shown in Table I.

Another major source of error comes from decoherence effects. We compare the experimental data to a simple phenomenological error model, i.e., the system undergoes uncorrelated dephasing channel, parametrized with a set of phase flip error probabilities $\left\{p_{i}\right\}_{i=1,2,3,4}$ per evolution time step $t_{0}$. The density matrix $\hat{\rho}$ is then, at each evolution step, subjected to the composition of the error channels $\mathcal{E}_{i}$ for each qubit [47]:

$$
\hat{\rho} \rightarrow \mathcal{E}_{4} \circ \mathcal{E}_{3} \circ \mathcal{E}_{2} \circ \mathcal{E}_{1}(\hat{\rho}),
$$

where

$$
\mathcal{E}_{i}(\hat{\rho})=\left(1-p_{i}\right) \hat{\rho}+p_{i} \hat{\sigma}_{i}^{z} \hat{\rho} \hat{\sigma}_{i}^{z} .
$$

with $p_{i}=\left(1-e^{-t_{0} / T_{2, i}}\right) / 2$ (see Fig. 5 for the values of $T_{2, i}$ ). The results are presented in Table I. The results indicate that, with decoherence effects taken into account, the discrepancy between theoretical and experimental data for $g=1, h=0$ is expected to be larger than that of the other two cases, consistent with the experiment data.

In summary, we conclude that $\mathrm{rf}$ inhomogeneity and decoherence effects are two major sources of errors.

\section{APPENDIX D: UNIT OF TIME $t$}

Our model Hamiltonian is actually written as $\hat{H}=$ $\sum_{i}\left(-J \hat{\sigma}_{i}^{z} \hat{\sigma}_{i+1}^{z}+g \hat{\sigma}_{i}^{x}+h \hat{\sigma}_{i}^{z}\right)$, where we automatically set $J=1$ in the main text, and we choose the natural unit $\hbar=1$ throughout. So our time $t$ is in fact in the unit of $\hbar / J$.

\section{APPENDIX E: NORMALIZATION CONDITION FOR THE ENTANGLEMENT ENTROPY AND OTOC RELATION}

The relationship between the growth of the second Rényi entropy after a quench and the OTOCs at equilibrium is given in Ref. [10]. For a system at infinite temperature, we quench it with any operation $\hat{O}$ at $t=0$. So the density matrix at time $t$ is $\hat{\rho}(t)=e^{-i \hat{H} t} \hat{O} \hat{\mathbf{1}} \hat{O}^{\dagger} e^{i \hat{H} t}$. Then we study the second entanglement Rényi entropy between the subregion $\mathcal{B}$, and the rest is denoted as $\mathcal{A}$. The reduced density matrix is $\hat{\rho}_{\mathcal{A}}(t)=\operatorname{Tr}_{\mathcal{B}} \hat{\rho}(t)$, which gives us the entropy $S_{\mathcal{A}}^{(2)}(t)=-\log \operatorname{Tr}_{\mathcal{A}}\left[\hat{\rho}_{\mathcal{B}}(t)^{2}\right]$. The growth of entanglement is related to the OTOCs via

$$
\exp \left(-S_{\mathcal{A}}^{(2)}\right)=\sum_{\hat{M} \in \mathcal{B}}\langle\hat{M}(t) \hat{V}(0) \hat{M}(t) \hat{V}(0)\rangle_{\beta=0}
$$

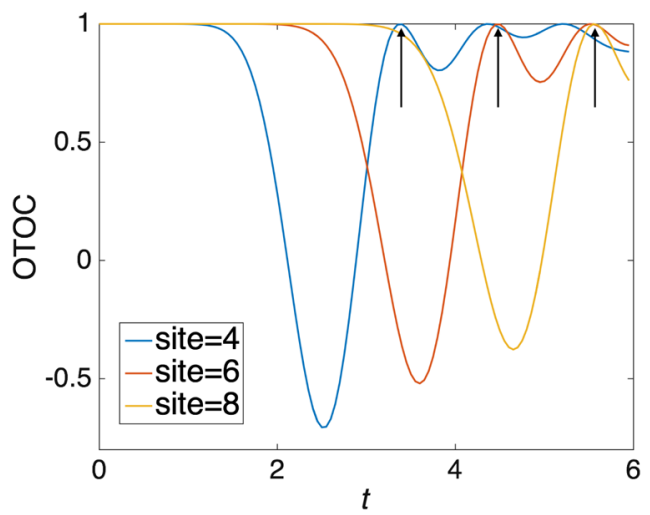

FIG. 9. Numerical results of OTOCs for the integrable case. The arrows denote the revival time, which approximately linearly increases with respect to the distance between operators. Here, we choose $\hat{A}=\hat{\sigma}_{z}^{1}$ on the first site and $\hat{B}=\hat{\sigma}_{z}^{n}$ on the final site. The parameters are $g=1, h=0$.

where the summation is taken over a complete set of operators in $\mathcal{B}$ and $\hat{V}=\hat{O} \hat{O}^{\dagger}$. Here, we should choose the following normalization condition: $\sum_{\hat{M} \in B} M_{i j} M_{l m}=$ $\delta_{i m} \delta_{l j}, \operatorname{Tr}\left[\hat{O} \hat{O}^{\dagger}\right]=\hat{\mathbf{1}}$.

Here, we quench the first site and take the first three sites as the subsystem $\mathcal{A}$ and the fourth site as the subsystem $\mathcal{B}$, as marked in Fig. 1(b) of the main text. Hence, we choose $\hat{O}=\left(\hat{\mathbf{1}}+\hat{\sigma}_{1}^{x}\right) / 2^{(D+1) / 2}(D=4$ is the total number of sites $)$. The complete set of operators in the subsystems $\mathcal{B}$ can be taken as $\hat{\sigma}_{4}^{\alpha} / \sqrt{2}$, where $\alpha=0, x, y, z$ and $\hat{\sigma}^{0}=\hat{\mathbf{1}}$. By summing over the measured data with the conventions above, we can get the points in Fig. 3 of the main text. The theoretical curves are obtained by directly computing entanglement entropy from the density matrix.

\section{APPENDIX F: REVIVAL TIME OF OTOC AND THE DISTANCE BETWEEN THE OPERATORS}

As we see from Fig. 2 of the main text, for the integrable case, the OTOCs will increase back around their initial values at some time. The revival time in fact depends on the spatial distance between the two operators, as depicted in Fig. 9. That is, the larger the distance, the later the revival happens. From the relationship between the growth of the second Rényi entropy after a quench and the OTOCs at equilibrium given in Ref. [10], we know that it will take longer time for the entanglement entropy to decrease back after a local quench.

[1] A. I. Larkin, and Y. N. Ovchinnikov, Quasiclassical Method in the Theory of Superconductivity, Sov. Phys. JETP 28, 1200 (1969).

[2] A. Kitaev, in Proceedings of the Fundamental Physics Prize Symposium (2014), Vol. 10. 
[3] S. H. Shenker and D. Stanford, Black Holes and the Butterfly Effect, J. High Energy Phys. 03 (2014) 067.

[4] S. H. Shenker and D. Stanford, Multiple Shocks, J. High Energy Phys. 12 (2014) 046.

[5] S. H. Shenker and D. Stanford, Stringy Effects in Scrambling, J. High Energy Phys. 05 (2015) 132.

[6] J. Maldacena, S. H. Shenker, and D. Stanford, A Bound on Chaos, J. High Energy Phys. 08 (2016) 106.

[7] A. Kitaev, Proceedings of the KITP Program: Entanglement in Strongly-Correlated Quantum Matter (2015).

[8] J. Maldacena and D. Stanford, Remarks on the Sachdev-Ye-Kitaev Model, Phys. Rev. D 94, 106002 (2016).

[9] H. Shen, P. Zhang, R. Fan, and H. Zhai, Out-of-Time-Order Correlation at a Quantum Phase Transition, arXiv: 1608.02438.

[10] R. Fan, P. Zhang, H. Shen, and H. Zhai, Out-of-Time-Order Correlation for Many-Body Localization, Science Bulletin 62, 707 (2017).

[11] P. Hosur, X.-L. Qi, D. A. Roberts, and B. Yoshida, Chaos in Quantum Channels, J. High Energy Phys. 02 (2016) 004.

[12] Y. Huang, Y.-L. Zhang, and X. Chen, Out-of-Time-Ordered Correlator in Many-Body Localized Systems, Ann. Phys. (Berlin), DOI: 10.1002/andp.201600318, 2016.

[13] Y. Chen, Quantum Logarithmic Butterfly in Many Body Localization, arXiv:1608.02765.

[14] B. Swingle and D. Chowdhury, Slow Scrambling in Disordered Quantum Systems, Phys. Rev. B 95, 060201 (2017).

[15] R.-Q. He and Z.-Y. Lu, Characterizing Many-Body Localization by Out-of-Time-Ordered Correlation, Phys. Rev. B 95, 054201 (2017).

[16] E. L. Hahn, Spin Echoes, Phys. Rev. 80, 580 (1950).

[17] M. F. Andersen, A. Kaplan, and N. Davidson, Echo Spectroscopy and Quantum Stability of Trapped Atoms, Phys. Rev. Lett. 90, 023001 (2003).

[18] H. T. Quan, Z. Song, X. F. Liu, P. Zanardi, and C.-P. Sun, Decay of Loschmidt Echo Enhanced by Quantum Criticality, Phys. Rev. Lett. 96, 140604 (2006).

[19] A. Goussev, R. A. Jalabert, H. M. Pastawski, and D. A. Wisniacki, Loschmidt Echo and Time Reversal in Complex Systems, Phil. Trans. R. Soc. A 374, 20150383 (2016).

[20] B. Swingle, G. Bentsen, M. Schleier-Smith, and P. Hayden, Measuring the Scrambling of Quantum Information, Phys. Rev. A 94, 040302 (2016).

[21] G. Zhu, M. Hafezi, and T. Grover, Measurement of ManyBody Chaos Using a Quantum Clock, Phys. Rev. A 94, 062329 (2016).

[22] N. Y. Yao, F. Grusdt, B. Swingle, M. D. Lukin, D. M. Stamper-Kurn, J. E. Moore, and E. A. Demler, Interferometric Approach to Probing Fast Scrambling, arXiv: 1607.01801.

[23] I. Danshita, M. Hanada, and M. Tezuka, Creating and Probing the Sachdev-Ye-Kitaev Model with Ultracold Gases: Towards Experimental Studies of Quantum Gravity, arXiv:1606.02454.

[24] R. P. Feynman, Simulating Physics with Computers, Int. J. Theor. Phys. 21, 467 (1982).
[25] S. Lloyd, Universal Quantum Simulators, Science 273, 1073 (1996).

[26] S. G. Schirmer, H. Fu, and A. I. Solomon, Complete Controllability of Quantum Systems, Phys. Rev. A 63, 063410 (2001).

[27] D. W. Leung, I. L. Chuang, F. Yamaguchi, and Y. Yamamoto, Efficient Implementation of Coupled Logic Gates for Quantum Computation, Phys. Rev. A 61, 042310 (2000).

[28] O. Gühne and G. Tóth, Entanglement Detection, Phys. Rep. 474, 1 (2009).

[29] D. A. Roberts, D. Stanford, and L. Susskind, Localized Shocks, J. High Energy Phys. 03 (2015) 051.

[30] M. Blake, Universal Charge Diffusion and the Butterfly Effect in Holographic Theories, Phys. Rev. Lett. 117, 091601 (2016).

[31] D. A. Roberts and B. Swingle, Lieb-Robinson Bound and the Butterfly Effect in Quantum Field Theories, Phys. Rev. Lett. 117, 091602 (2016).

[32] E. H. Lieb, and D. W. Robinson, Statistical Mechanics (Springer, New York, 1972), pp. 425-431.

[33] S. Debnath, N. M. Linke, C. Figgatt, K. A. Landsman, K. Wright, and C. Monroe, Demonstration of a Small Programmable Quantum Computer with Atomic Qubits, Nature (London) 536, 63 (2016).

[34] R. Barends et al., Superconducting Quantum Circuits at the Surface Code Threshold for Fault Tolerance, Nature (London) 508, 500 (2014).

[35] J. Kelly et al., State Preservation by Repetitive Error Detection in a Superconducting Quantum Circuit, Nature (London) 519, 66 (2015).

[36] C. Monroe and J. Kim, Scaling the Ion Trap Quantum Processor, Science 339, 1164 (2013).

[37] J. G. Bohnet, B. C. Sawyer, J. W. Britton, M. L. Wall, A. M. Rey, M. Foss-Feig, and J. J. Bollinger, Quantum Spin Dynamics and Entanglement Generation with Hundreds of Trapped Ions, Science 352, 1297 (2016).

[38] A. D. Córcoles, E. Magesan, S. J. Srinivasan, A. W. Cross, M. Steffen, J. M. Gambetta, and J. M. Chow, Demonstration of a Quantum Error Detection Code Using a Square Lattice of Four Superconducting Qubits, Nat. Commun. 6, 6979 (2015).

[39] J. M. Gambetta, J. M. Chow, and M. Steffen, Building Logical Qubits in a Superconducting Quantum Computing System, Quantum Inf. Comput. 3, 2 (2017).

[40] M. Garttner, J. G. Bohnet, A. Safavi-Naini, M. L. Wall, J. J. Bollinger, and A. M. Rey, Measuring Out-of-Time-Order Correlations and Multiple Quantum Spectra in a Trapped Ion Quantum Magnet, Nat. Phys., DOI: 10.1038/nphys4119 (2017).

[41] X. Peng, Z. Luo, W. Zheng, S. Kou, D. Suter, and J. Du, Experimental Implementation of Adiabatic Passage between Different Topological Orders, Phys. Rev. Lett. 113, 080404 (2014).

[42] M. H. Levitt and L. Di Bari, Steady State in Magnetic Resonance Pulse Experiments, Phys. Rev. Lett. 69, 3124 (1992).

[43] L. M. Vandersypen and I. L. Chuang, NMR Techniques for Quantum Control and Computation, Rev. Mod. Phys. 76, 1037 (2005). 
[44] C. A. Ryan, C. Negrevergne, M. Laforest, E. Knill, and R. Laflamme, Liquid-State Nuclear Magnetic Resonance as a Testbed for Developing Quantum Control Methods, Phys. Rev. A 78, 012328 (2008).

[45] J. Li, J. Cui, R. Laflamme, and X. Peng, Selective-PulseNetwork Compilation on a Liquid-State Nuclear-MagneticResonance System, Phys. Rev. A 94, 032316 (2016).
[46] N. Khaneja, T. Reiss, C. Kehlet, T. Schulte-Herbrüggen, and S. J. Glaser, Optimal Control of Coupled Spin Dynamics: Design of NMR Pulse Sequences by Gradient Ascent Algorithms, J. Magn. Reson. 172, 296 (2005).

[47] M. A. Nielsen and I. L. Chuang, Quantum Computation and Quantum Information (Cambridge University Press, Cambridge, England, 2010). 